\title{
Determination of Dissociation Constants of Some Novel Tetrahydropyrimidine Derivatives in Mixed Organic-Water System by Simple pH Measurement
}

\author{
Shipra Baluja*, Kapil D. Bhesaniya and Ashish B. Patel \\ Physical Chemistry Laboratory, Department of Chemistry, Saurashtra University, Rajkot-360005 (Gujarat), \\ India. \\ Email: shipra_baluja@rediffmail.com
}

\begin{abstract}
Some tetrahydropyrimidine derivatives have been synthesized and their structures have been confirmed by IR, ${ }^{1} \mathrm{H}$ NMR, ${ }^{13} \mathrm{C}$ NMR and mass spectral data. The dissociation constants of these derivatives of tetrahydropyrimidine have been measured by Calvin Bjerrum $\mathrm{pH}$ titration method in DMF-water (60: $40 \mathrm{v} / \mathrm{v}$ ) system at $303.15 \mathrm{~K}$. It is observed that dissociation constant depends on substituent groups present in the compounds.
\end{abstract}

Keywords: Tetrahydropyrimidine derivatives, dissociation constant, DMF-water system, Calvin Bjerrum pH titration method

\section{Introduction}

Synthesis of tetrahydropyrimidine and its derivatives is of high interest in organic chemistry. The pyrimidine fragment is present in various biologically active compounds, many of which are used therapeutically [1-3]. Thus, much attention has been paid to derivatives of pyrimidines, including their hydrogenation products. This class of compounds possesses a wide range of biological and pharmacological properties such as antidepressant [4], calcium antagonist [5,6], antitumor [7], antitubercular [8] anti-inflammatory [9, 10], antibacterial and antifungal effects [11, 12], analgesic [13,14], antioxidant [15], etc.

Therefore, the synthesis of tetrahydropyrimidine derivatives continues to attract much interest in organic chemistry. These applications prompted us to study their dissociation constant which is an important parameter required in various fields such as pharmaceutical, chemical, biological and environmental research. Further, it helps to study the transport of drugs into cells and for optimizing drug delivery [16]. A literature survey shows that various workers have studied the dissociation constant of organic compounds $[17,18]$. In the present work, the dissociation constant of synthesized tetrahydropyrimidine derivatives are studied in dimethyl formamide-water mixture at $303.15 \mathrm{~K}$ by Calvin-Bjerrum $p H$ titration technique.

\section{$2 \quad$ Experimental}

\subsection{Materials}

Different aromatic aldehydes, malenonitrile, and guanidine hydrochloride used in the synthesis were of AR grade and were purchased from Spectrochem Pvt. Ltd. (Mumbai, India). Sodium ethoxide was prepared in our laboratory by dissolving sodium metal in ethanol $(99.5 \% \mathrm{~V} / \mathrm{V})$ (Baroda Chemical Industries Ltd. Vadodara). Sodium nitrate $\left(\mathrm{NaNO}_{3}\right)$ (CAS No.: 7631-99-4), Nitric acid $\left(\mathrm{HNO}_{3}\right)(\mathrm{CAS}$ No.: 7697-37-2) and Sodium hydroxide (NaOH) (CAS No.: 1310-73-2) were purchased from SD FINE CHEM. Ltd (Vadodara-India). The solvent DMF used in the present work was AR grade supplied by Spectrochem Pvt. Ltd. (Mumbai, India) and was purified according to the standard procedure [19]. The purity of solvent was checked by GC-MS (SHIMADZU-Model No.-QP-2010) and was found to be greater than $99.8 \%$. 


\subsection{Synthesis}

To an equimolar mixture of substituted aldehyde, malenonitrile, guanidine hydrochloride and freshly prepared ethanolic sodium ethoxide solution was added and the mixture was refluxed for $12 \mathrm{~h}$. The progress of the reaction was monitored with the aid of analytical thin layer chromatography (Performed on aluminum coated TLC plates Gel 60 F254 (E. Merck)). After the completion of reaction, the reaction mixture was poured into crushed ice. The solution was neutralized with aqueous $\mathrm{HCl}$ solution and the product was extracted using chloroform. The solvent was removed under reduced pressure and the resulting compound was crystallized using chloroform. The synthesized compounds were purified. The reaction scheme is given in Fig. 1.

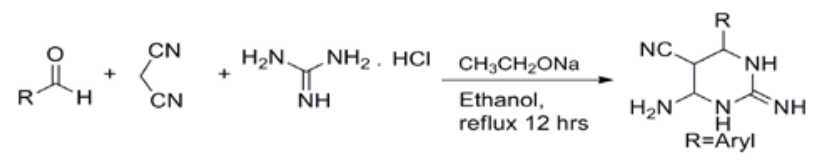

Figure 1. Reaction scheme of tetrahydropyrimidine derivatives

Spectroscopic study of all the synthesized compounds was done by IR, ${ }^{1} \mathrm{H}$ NMR and mass spectrometry. IR spectra were recorded on KBr discs, using FT-IR Model No.-8400 (Shimadzu) spectrophotometer. ${ }^{1} \mathrm{H}-\mathrm{NMR}$ spectra were taken on a Bruker Avance II-400. In all cases, NMR spectra were obtained in DMSO- $\mathrm{d}_{6}$ using TMS as an internal standard The NMR signals are reported in $\delta \mathrm{ppm}$. Mass spectra were obtained using direct inlet probe on a GCMS-QP-2010 mass spectrometer. The melting points of all the synthesized compounds were determined in open capillary tubes and were uncorrected.

\subsection{Dissociation Constant Measurement}

$0.1 \mathrm{M}$ solutions of all the compounds were prepared in DMF. These solutions were retained at the desired temperature. The stock solutions of desired concentration of $\mathrm{HNO}_{3}, \mathrm{NaOH}$ and $\mathrm{NaNO}_{3}$ required for titrations were prepared in Milli-Q water (Millipore Pvt. Lt. Bangalore, India). An electrical balance (Mettler Toledo AB204-S) with an accuracy of $\pm 0.1 \mathrm{mg}$ was used.

The Calvin Bjerrum pH titration method [20,21] was used to determine dissociation constants of all the synthesized compounds. For this, two sets of solution were prepared.

(i) $2.0 \mathrm{ml} \mathrm{HNO} 3(0.1 \mathrm{M})+4.0 \mathrm{ml}$ water $+30.0 \mathrm{ml}(\mathrm{DMF})+4.0 \mathrm{ml} \mathrm{NaNO}{ }_{3}(1.0 \mathrm{M})$.

(ii) $2.0 \mathrm{ml} \mathrm{HNO}_{3}(0.1 \mathrm{M})+4.0 \mathrm{ml}$ water $+28.0 \mathrm{ml}(\mathrm{DMF})+2.0 \mathrm{ml}$ compound solution $(0.1 \mathrm{M})+4.0$ $\mathrm{ml} \mathrm{NaNO}$ (1.0 M).

At temperature $303.15 \mathrm{~K}$, both sets of solutions were titrated against $0.5 \mathrm{M} \mathrm{NaOH}$ and corresponding $\mathrm{pH}$ was noted by Systronic $\mathrm{pH}$ meter (Model No. EQ-664). The Systronic glass electrode and a saturated calomel electrode were used as indicator and reference electrodes respectively. Before measurement, the $\mathrm{pH}$ meter was calibrated with buffer solution of known $\mathrm{pH}(\mathrm{pH} 4.0$ and 9.18).

However, in the present study, DMF-water $(60: 40 \mathrm{v} / \mathrm{v})$ solvent systems are used, so the following [22] relation was used for $\mathrm{pH}$ correction.

$$
-\log \left[H^{+}\right]=p H+\log f+\log U_{H}^{0}
$$

where $f$ is the activity coefficient of the hydrogen ions in the solvent mixtures under consideration at the same temperature and ionic strength and $U_{H}^{0}$ is a correction factor at zero ionic strength, which depends only on the solvent composition and temperature.

The constant temperature was adjusted to $0.05 \mathrm{~K}$ by circulating the thermo stated water through the outer jacket of the vessel.

\section{$3 \quad$ Results and Discussion}

Ten compounds (PAB-100 to PAB-110) are synthesized and their physical properties are given in Table 1. IR, ${ }^{1} \mathrm{H}$ NMR and ${ }^{13} \mathrm{C}$ NMR spectrum for compound PAB-107 are given in Figures 2, 3 and 4 respectively. 
Table 1. Physical properties of synthesized tetrahydropyrimidine derivatives.

\begin{tabular}{|c|c|c|c|c|c|}
\hline Comp. Code & $\mathbf{R}$ & $\begin{array}{c}\text { Molecular } \\
\text { formula }\end{array}$ & $\begin{array}{c}\text { Molecular } \\
\text { weight }\end{array}$ & $\begin{array}{l}\text { Yield } \\
(\%) \\
\end{array}$ & $\mathbf{R}_{\mathrm{f}}{ }^{*}$ \\
\hline$P A B-101$ & $\mathrm{C}_{6} \mathrm{H}_{5}^{-}$ & $\mathrm{C}_{11} \mathrm{H}_{13} \mathrm{~N}_{5}$ & 215.25 & 78 & 0.68 \\
\hline$P A B-102$ & $\mathrm{C}_{6} \mathrm{H}_{4}-\mathrm{CH}=\mathrm{CH}-$ & $\mathrm{C}_{13} \mathrm{H}_{15} \mathrm{~N}_{5}$ & 241.29 & 60 & 0.73 \\
\hline$P A B-103$ & $3-\mathrm{Cl}, \mathrm{C}_{6} \mathrm{H}_{4^{-}}$ & $\mathrm{C}_{11} \mathrm{H}_{12} \mathrm{ClN}_{5}$ & 249.70 & 68 & 0.44 \\
\hline$P A B-104$ & $4-\mathrm{Cl}, \mathrm{C}_{6} \mathrm{H}_{4^{-}}$ & $\mathrm{C}_{11} \mathrm{H}_{12} \mathrm{ClN}_{5}$ & 249.70 & 66 & 0.46 \\
\hline$P A B-105$ & $4-\mathrm{F}, \mathrm{C}_{6} \mathrm{H}_{4^{-}}$ & $\mathrm{C}_{12} \mathrm{H}_{15} \mathrm{FN}_{5}$ & 233.24 & 62 & 0.52 \\
\hline$P A B-106$ & $4-\mathrm{OCH}_{3}, \mathrm{C}_{6} \mathrm{H}_{4^{-}}$ & $\mathrm{C}_{12} \mathrm{H}_{15} \mathrm{~N}_{5} \mathrm{O}$ & 245.28 & 84 & 0.42 \\
\hline$P A B-107$ & $3-\mathrm{NO}_{2}, \mathrm{C}_{6} \mathrm{H}_{4^{-}}$ & $\mathrm{C}_{11} \mathrm{H}_{12} \mathrm{~N}_{6} \mathrm{O}_{2}$ & 260.25 & 60 & 0.62 \\
\hline$P A B-108$ & $3-\mathrm{OCH}_{3}, 4-\mathrm{OH} \mathrm{C} \mathrm{H}_{4} \mathrm{H}^{-}$ & $\mathrm{C}_{12} \mathrm{H}_{15} \mathrm{~N}_{5} \mathrm{O}_{2}$ & 261.28 & 74 & 0.34 \\
\hline$P A B-109$ & $4\left(\alpha-\mathrm{C}_{4} \mathrm{H}_{3} \mathrm{O}\right)-$ & $\mathrm{C}_{9} \mathrm{H}_{11} \mathrm{~N}_{5} \mathrm{O}$ & 205.22 & 82 & 0.36 \\
\hline$P A B-110$ & $4-\mathrm{OH}-\mathrm{C}_{6} \mathrm{H}_{4^{-}}$ & $\mathrm{C}_{11} \mathrm{H}_{13} \mathrm{~N}_{5} \mathrm{O}$ & 231.25 & 56 & 0.30 \\
\hline
\end{tabular}

*Mobile phase: (9.5:0.5-Dichloromethane : methanol)

\subsection{Spectral Data}

2,4-diamino-6-phenyl-1,4,5,6-tetrahydropyrimidine-5-carbonitrile (PAB-101). mp. $141-143^{\circ} \mathrm{C}$;

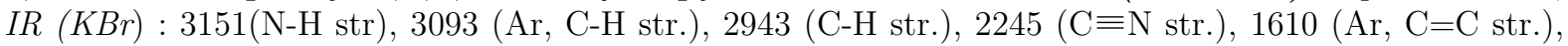
1519 (Ar, C=C str.), 1512(Ar, C=C str.), 1490 (C-H bend.), 1427(C-H bend.), 1377 (C-H bend.), 1265 (C-C str.) $\mathrm{cm}^{-1} ;{ }^{1} H \mathrm{NMR}\left(400 \mathrm{MHz}, \mathrm{DMSO}-\mathrm{d}_{6}\right): \delta \mathrm{ppm} 2.59(\mathrm{~s}, 1 \mathrm{H},-\mathrm{CH}) 3.22-3.36(\mathrm{t}, 1 \mathrm{H},-\mathrm{CH}), 4.09-$ $4.12(\mathrm{~d}, J=9.81 \mathrm{~Hz} 1 \mathrm{H},-\mathrm{CH}), 6.29\left(\mathrm{~s}, 2 \mathrm{H}, \mathrm{NH}_{2}\right), 6.66\left(\mathrm{~s}, 2 \mathrm{H}, \mathrm{NH}_{2}\right), 8.38-8.42(\mathrm{~m}, 5 \mathrm{H}, \mathrm{Ar}-\mathrm{H}), 8.64(\mathrm{~s}$, $1 \mathrm{H},-\mathrm{NH}) . \quad M S: \mathrm{m} / z=215[\mathrm{M}]^{+}$.

2,4-diamino-6-styryl)-1,4,5,6-tetrahydropyrimidine-5-carbonitrile(PAB-102). mp. $112-114^{\circ} \mathrm{C}$; IR ( KBr $): 3161(\mathrm{~N}-\mathrm{H}$ str), 3021 ( $\mathrm{CH}=\mathrm{CH}$ str.), 3091 (Ar, C-H str.), 2952 (C-H str.), 2267 ( $\mathrm{C} \equiv \mathrm{N}$ str.), 1609 (Ar, $\mathrm{C}=\mathrm{C}$ str.), 1515 (Ar, $\mathrm{C}=\mathrm{C}$ str.), 1508 (Ar, $\mathrm{C}=\mathrm{C}$ str.), 1481(C-H bend.), 1425 (C-H bend.), 1379 (C-H bend.), 1252 (C-C str.) $\mathrm{cm}^{-1} ;{ }^{1} H \mathrm{NMR}$ (400 MHz, DMSO-d 6 ) : $\delta$ ppm 2.55 (s, 1H, -CH) 3.29$3.37(\mathrm{t}, 1 \mathrm{H},-\mathrm{CH}), 4.05-4.18(\mathrm{~d}, J=9.79 \mathrm{~Hz} 1 \mathrm{H},-\mathrm{CH}), 5.04-5.07$ (dd, J=9.79 Hz 1H, $-\mathrm{CH}), 5.04-5.07$ $(\mathrm{dd}, \mathrm{J}=8.24 \mathrm{~Hz}), 6.32\left(\mathrm{~s}, 2 \mathrm{H}, \mathrm{NH}_{2}\right), 6.6\left(\mathrm{~s}, 2 \mathrm{H}, \mathrm{NH}_{2}\right), 8.34-8.39(\mathrm{~m}, 5 \mathrm{H}, \mathrm{Ar}-\mathrm{H}), 8.74(\mathrm{~s}, 1 \mathrm{H},-\mathrm{NH}), M S$ : $\mathrm{m} / z=241[\mathrm{M}]^{+}$.

2,4-diamino-6-(3-chlorophenyl)-1,4,5,6-tetrahydropyrimidine-5-carbonitrile (PAB-103). mp.

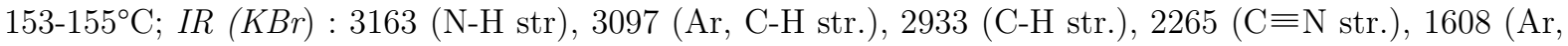
$\mathrm{C}=\mathrm{C}$ str.), 1514 (Ar, $\mathrm{C}=\mathrm{C}$ str.), 1502(Ar, $\mathrm{C}=\mathrm{C}$ str.), 1489 (C-H bend.), 1421 (C-H bend.), 1371 (C-H bend.), 1255 (C-C str.), 744 (C-Cl) $\mathrm{cm}^{-1} ;{ }^{1} H \mathrm{NMR}\left(400 \mathrm{MHz}, \mathrm{DMSO}-d_{6}\right): \delta \mathrm{ppm} 2.61$ (s, 1H, -CH) 3.30$3.34(\mathrm{t}, 1 \mathrm{H},-\mathrm{CH}), 4.07-4.09(\mathrm{~d}, J=9.78 \mathrm{~Hz} 1 \mathrm{H},-\mathrm{CH}), 6.21\left(\mathrm{~s}, 2 \mathrm{H}, \mathrm{NH}_{2}\right), 6.68\left(\mathrm{~s}, 2 \mathrm{H}, \mathrm{NH}_{2}\right), 7.82-7.87(\mathrm{t}$, $1 \mathrm{H}, \mathrm{Ar}-\mathrm{H}), 8.39-8.42(\mathrm{~d}, 1 \mathrm{H}, \mathrm{Ar}-\mathrm{H}), 8.44-8.49(\mathrm{~m}, 1 \mathrm{H}, \mathrm{Ar}-\mathrm{H}), 8.64(\mathrm{~s}, 1 \mathrm{H},-\mathrm{NH}), 8.81(\mathrm{~s}, 1 \mathrm{H}, \mathrm{Ar}-\mathrm{H}) . M S$ : $\mathrm{m} / z=249[\mathrm{M}]^{+}$.

2,4-diamino-6-(4-chlorophenyl)-1,4,5,6-tetrahydropyrimidine-5-carbonitrile(PAB-104). mp.

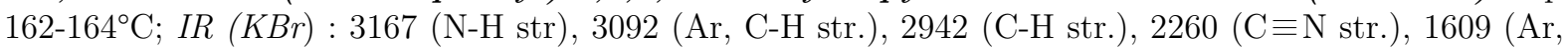
$\mathrm{C}=\mathrm{C}$ str.), 1508 (Ar, $\mathrm{C}=\mathrm{C}$ str.), 1518 (Ar, $\mathrm{C}=\mathrm{C}$ str.), 1481 (C-H bend.), 1422 (C-H bend.), 1376 (C-H bend.), 1250 (C-C str.), 742 (C-Cl) $\mathrm{cm}^{-1} ;{ }^{1} H$ NMR (400 MHz, DMSO-d $\left.{ }_{6}\right): \delta$ ppm 2.63 (s, 1H, -CH) 3.24$3.29(\mathrm{t}, 1 \mathrm{H},-\mathrm{CH}), 4.12-4.15(\mathrm{~d}, J=9.78 \mathrm{~Hz} 1 \mathrm{H},-\mathrm{CH}), 6.21\left(\mathrm{~s}, 2 \mathrm{H}, \mathrm{NH}_{2}\right), 6.63\left(\mathrm{~s}, 2 \mathrm{H}, \mathrm{NH}_{2}\right), 7.82-7.87$ (dd, $2 \mathrm{H}$, Ar- H) $, 8.39-8.42(\mathrm{dd}, 2 \mathrm{H}, \mathrm{Ar}-\mathrm{H}), 8.64(\mathrm{~s}, 1 \mathrm{H},-\mathrm{NH}) . M S: \mathrm{m} / z=249[\mathrm{M}]^{+}$.

2,4-diamino-6-(4-fluorophenyl)-1,4,5,6-tetrahydropyrimidine-5-carbonitrile (PAB-105). mp. 128-130 ${ }^{\circ} \mathrm{C}$; IR ( $\left.\mathrm{KBr}\right): 3170$ (N-H str), 3094 (Ar, C-H str.), 2949 (C-H str.), 2250 (C $\equiv \mathrm{N}$ str.), 1622 (Ar, $\mathrm{C}=\mathrm{C}$ str.), 1510 (Ar, $\mathrm{C}=\mathrm{C}$ str.), 1520 (Ar, $\mathrm{C}=\mathrm{C}$ str.), 1486 (C-H bend.), 1422 (C-H bend.), 1371 (C-H bend.), 1259 (C-C str.), 1065 (C-F) $\mathrm{cm}^{-1} ;{ }^{1} \mathrm{H} N M R$ (400 MHz, DMSO-d d $_{6}: \delta \mathrm{ppm} 2.60$ (s, 1H, -CH), 3.25-3.28 (t, $1 \mathrm{H},-\mathrm{CH}), 4.15-4.19(\mathrm{~d}, J=9.78 \mathrm{~Hz} 1 \mathrm{H},-\mathrm{CH}), 6.21$ (s, $\left.2 \mathrm{H}, \mathrm{NH}_{2}\right), 6.69\left(\mathrm{~s}, 2 \mathrm{H}, \mathrm{NH}_{2}\right), 7.96-$ $7.99(\mathrm{dd}, 2 \mathrm{H}, \mathrm{Ar}-\mathrm{H}), 8.46-8.49(\mathrm{dd}, 2 \mathrm{H}, \mathrm{Ar}-\mathrm{H}), 8.66(\mathrm{~s}, 1 \mathrm{H},-\mathrm{NH}), M S: \mathrm{m} / z=233[\mathrm{M}]^{+}$.

2,4-diamino-6-(4-methoxyphenyl)-1,4,5,6-tetrahydropyrimidine-5-carbonitrile (PAB-106). mp. $116-118^{\circ} \mathrm{C} ; \operatorname{IR}(\mathrm{KBr}): 3170$ (N-H str), 3094 (Ar, C-H str.), 29497 (C-H str.), 2250 (C $\equiv \mathrm{N}$ str.), 1619 (Ar, C=C str.), 1508 (Ar, C=C str.), 1478 (C-H bend.), 1426 (C-H bend.), 1368 (C-H bend.), 1254 (C-C str.), 1157 (C-O-C) $\mathrm{cm}^{-1} ;{ }^{1} H$ NMR $\left(400 \mathrm{MHz}, \mathrm{DMSO}_{6}\right): \delta \mathrm{ppm} 2.67(\mathrm{~s}, 1 \mathrm{H},-\mathrm{CH}), 3.71\left(\mathrm{~s}, 3 \mathrm{H},-\mathrm{OCH}_{3}\right)$, 
3.24-3.29 (t, 1H, -CH), 4.10-4.13 (d, $J=9.75 \mathrm{~Hz} 1 \mathrm{H},-\mathrm{CH}), 6.23\left(\mathrm{~s}, 2 \mathrm{H}, \mathrm{NH}_{2}\right), 6.67\left(\mathrm{~s}, 2 \mathrm{H}, \mathrm{NH}_{2}\right), 7.83-$ 7.87 (dd, 2H, Ar- H), 8.39-8.43 (dd, 2H, Ar-H), 8.54 (s, 1H, Ar-H). MS: m/z=245[M] ${ }^{+}$.

2,4-diamino-6-(3-nitrophenyl)-1,4,5,6-tetrahydropyrimidine-5-carbonitrile (PAB-107). mp.

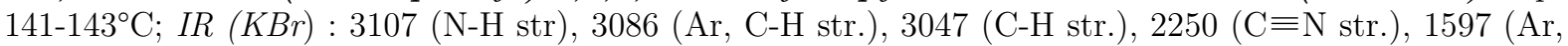
$\mathrm{C}=\mathrm{C}$ str.), 1527 (Ar, C=C str.), 1512 (Ar, C=C str.), 1479 (C-H bend.), 1427 (C-H bend.), 1315(C-H bend.), 1217 (C-C str.) $\mathrm{cm}^{-1} ;{ }^{1} H$ NMR (400 MHz, DMSO-d $\left.{ }_{6}\right): \delta$ ppm $2.56(\mathrm{~s}, 1 \mathrm{H},-\mathrm{CH}) 3.20-3.34$ (t, 1H, $-\mathrm{CH}), 4.06-4.08(\mathrm{~d}, J=9.80 \mathrm{~Hz} 1 \mathrm{H},-\mathrm{CH}), 6.24\left(\mathrm{~s}, 2 \mathrm{H}, \mathrm{NH}_{2}\right), 6.60\left(\mathrm{~s}, 2 \mathrm{H}, \mathrm{NH}_{2}\right), 7.84-7.88$ (t, $\left.1 \mathrm{H}, \mathrm{Ar}-\mathrm{H}\right)$, 8.35-8.37 (d, 1H, Ar-H), 8.44-8.47 (m, 1H, Ar-H), 8.63 (s, 1H, -NH), 8.83(s, 1H, Ar-H). 13C NMR (100 $\mathrm{MHz}, \mathrm{DMSO}): \delta \mathrm{ppm} 38.96,39.17,58.50,119.68,123.40,128.37,133.56,138.54,164.05 ;{ }^{13} \mathrm{C} \mathrm{NMR}$ $\left(400 \mathrm{MHz}, \mathrm{DMSO}-d_{6}\right) \delta(\mathrm{ppm}): 164.05,148.99,138.54,133.56,129.06,128.37,123.40,78.94,78.74,78.41$, 58.80, $M S: \mathrm{m} / z=260[\mathrm{M}]^{+}$.

2,4-diamino-6-(4-hydroxy-3-methoxyphenyl)-1,4,5,6-tetrahydropyrimidine-5-carbonitrile (PAB-108). mp. 132-134 C; IR (KBr) : 3480 (OH str.), 3164 (N-H str), 3023 (Ar, C-H str.), 2947 (C-H str.), 2241 (C $\equiv \mathrm{N}$ str.), 1606 (Ar, $\mathrm{C}=\mathrm{C}$ str.), 1523 (Ar, C=C str.), 1538 (Ar, C=C str.), 1482 (C-H bend.), 1357 (C-H bend.), 1230 (C-C str.), 1078 (C-O-C str.) cm ${ }^{-1} ;{ }^{1} H ~ N M R$ (400 MHz, DMSO-d D $_{6}$ : $\delta$ ppm $2.50(\mathrm{~s}, 1 \mathrm{H},-\mathrm{CH}) 3.29-3.33(\mathrm{t}, 1 \mathrm{H},-\mathrm{CH}), 3.74\left(\mathrm{~s}, 3 \mathrm{H}, \mathrm{OCH}_{3}\right), 4.07-4.09(\mathrm{~d}, J=9.86 \mathrm{~Hz} 1 \mathrm{H},-\mathrm{CH})$, $4.89(\mathrm{~s}, 1 \mathrm{H},-\mathrm{OH}), 6.26\left(\mathrm{~s}, 2 \mathrm{H}, \mathrm{NH}_{2}\right), 6.65\left(\mathrm{~s}, 2 \mathrm{H}, \mathrm{NH}_{2}\right), 7.84-7.88$ (t, 1H, Ar- H), 8.35-8.37 (d, 1H, Ar-H), 8.45-8.49 (m, 1H, Ar-H), $8.69(\mathrm{~s}, 1 \mathrm{H},-\mathrm{NH}) . M S: \mathrm{m} / z=261[\mathrm{M}]^{+}$.

2,4-diamino-6-(furan-2-yl)-1,4,5,6-tetrahydropyrimidine-5-carbonitrile (PAB-109). mp. 72$74^{\circ} \mathrm{C}$; IR (KBr) : 3124 (N-H str), 3043 (Ar, C-H str.), 2922 (C-H str.), 2245 (C $\equiv \mathrm{N}$ str.), 1606 (Ar, C=C str.), 1552 (Ar, C=C str.), 1529 (Ar, C=C str.), 1456 (C-H bend.), 1394 (C-H bend.), 1330 (C-H bend.), 1230 (C-C str.), 1070 (C-O-C), 1022 (C-O-C str.) cm ${ }^{-1} ;{ }^{1} H ~ N M R ~\left(400 ~ M H z, D M S O-d_{6}\right): \delta$ ppm 2.57 (s, $1 \mathrm{H},-\mathrm{CH}) 3.32-3.35$ (t, $1 \mathrm{H},-\mathrm{CH}), 4.07-4.09$ ( d, $J=9.80 \mathrm{~Hz} 1 \mathrm{H},-\mathrm{CH}), 6.18\left(\mathrm{~s}, 2 \mathrm{H}, \mathrm{NH}_{2}\right), 6.64(\mathrm{~s}, 2 \mathrm{H}$, $\mathrm{NH}_{2}$ ), 7.84-7.88 (t, 1H, Ar- H), 8.35-8.37 (s, 1H, Ar-H), 8.45-8.49 (s, 1H, Ar-H), 8.71 (s, 1H, -NH). MS: $\mathrm{m} / z=205[\mathrm{M}]^{+}$.

2,4-diamino-6-(4-hydroxyphenyl)-1,4,5,6-tetrahydropyrimidine-5-carbonitrile (PAB-110). mp. 176-178 ${ }^{\circ}$; IR (KBr) : 3635 (OH str.), 3165(N-H str), 3088 (Ar, C-H str.), 2942 (C-H str.), 2250 (C $\equiv \mathrm{N}$ str.), 1619 (Ar, C=C str.), 1528 (Ar, C=C str.), 1510 (Ar, C=C str.), 1482 (C-H bend.), 1426 (C-H bend.), 1371 (C-H bend.), 1259 (C-C str.), 1210 (C-O) $\mathrm{cm}^{-1} ;{ }^{1} H$ NMR (400 MHz, DMSO-d $\left.{ }_{6}\right): \delta$ ppm $2.69(\mathrm{~s}, 1 \mathrm{H},-\mathrm{CH}), 3.29-3.32(\mathrm{t}, 1 \mathrm{H},-\mathrm{CH}), 4.15-4.18(\mathrm{~d}, J=9.78 \mathrm{~Hz} 1 \mathrm{H},-\mathrm{CH}), 4.64(\mathrm{~s}, 1 \mathrm{H}, \mathrm{OH}), 6.28(\mathrm{~s}$, $\left.2 \mathrm{H}, \mathrm{NH}_{2}\right), 6.69$ (s, 2H, $\mathrm{NH}_{2}$ ), 7.85-7.89 (dd, 2H, Ar- H), 8.46-8.50 (dd, 2H, Ar-H), 8.59 (s, 1H, -NH). MS: $\mathrm{m} / z=231[\mathrm{M}]^{+}$.

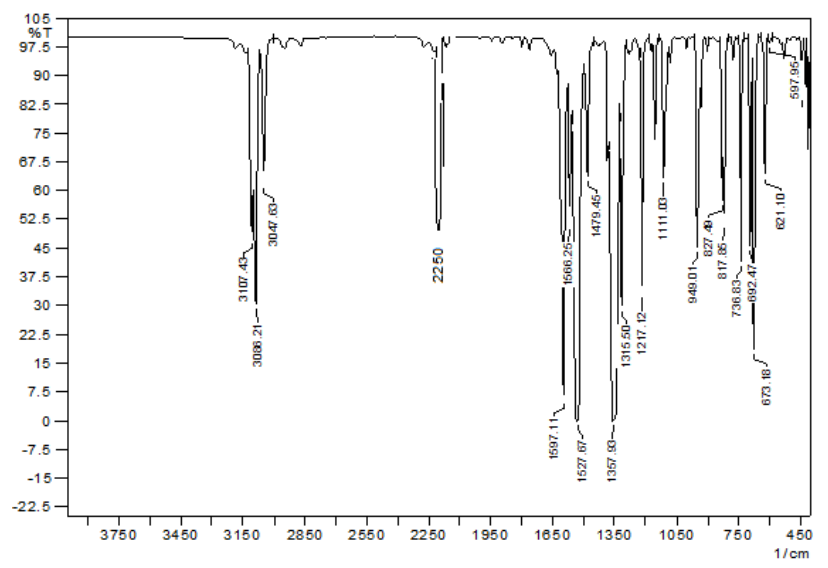

Figure 2. IR spectrum of PAB-107 


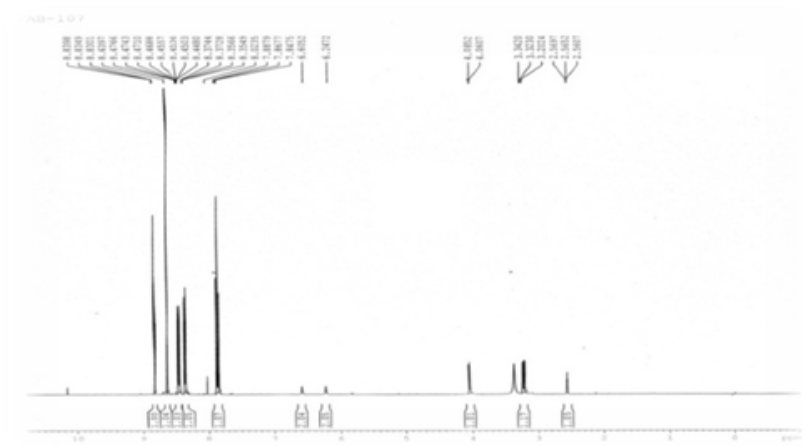

Figure 3. ${ }^{1} \mathrm{H}$ NMR spectrum of PAB-107

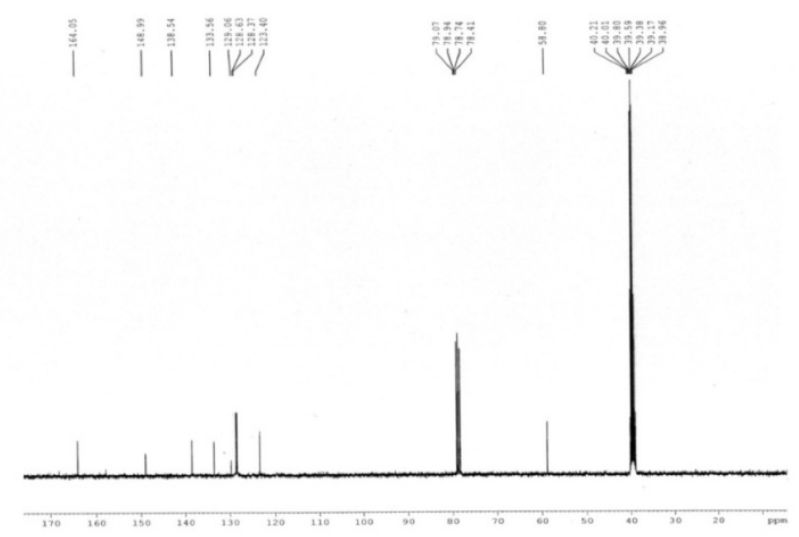

Figure 4. ${ }^{13} \mathrm{C}$ NMR spectrum of PAB-107

\subsection{Dissociation Constant Study}

Fig. 5 shows typical titration curves of the acid in the absence and presence of compound for PAB-101. It can be seen that for the same volume of $\mathrm{NaOH}$ added, the compound titration curves showed a lower $\mathrm{pH}$ value than the titration curve of free acid.

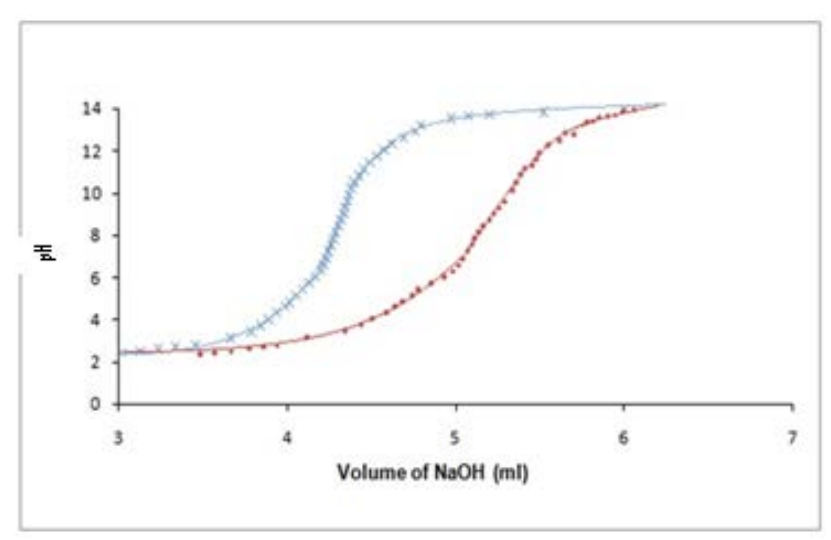

Figure 5. Plot of $\mathrm{pH}$ of acid (X) and acid + compound (ם) against volume of $\mathrm{NaOH}(V)$ for PAB-1 at $303.15 \mathrm{~K}$

From these titration curves, the average number of protons associated with the compound $\left(\overline{n_{H}}\right)$ can be calculated by using Irving and Rossotti equation [23]. 


$$
\overline{n_{H}}=Y-\left\{\left(V^{\prime \prime}-V^{\prime}\right)\left(N^{0}+E^{0}\right)\right\} /\left\{\left(V^{0}+V^{\prime}\right) T_{L}^{0}\right\}
$$

$\mathrm{V}^{\prime}$ and $\mathrm{V}^{\prime \prime}$ are the volume of alkali required at the same $\mathrm{pH}$ for both acid and compound titration curves respectively. $\mathrm{V}^{0}$ is the initial volume of the test solution. $\mathrm{N}^{0}, \mathrm{E}^{0}$ and $\mathrm{T}_{\mathrm{L}}^{0}$ are the initial concentration of the alkali, acid and compound respectively. $\mathrm{Y}$ is number of replaceable protons. For all the compounds (except PAB-108 and PAB-110), value of $\overline{n_{H}}$ is less than one; for PAB-108 and PAB110 , value of $\overline{n_{H}}$ is less than 2 , suggesting thereby presence of two replaceable protons in these two compounds.

The dissociation constants of all the compounds were evaluated by two methods; average and half integral methods. For average method, $p K_{1}^{H}$ values at $\overline{n_{H}}=0.5$ were evaluated for each compound (except PAB-108 and PAB-110) by using following equation:

$$
\log p K^{H}=p H+\log \overline{\left(n_{H}\right.} /\left(\overline{n_{H}}-1\right)
$$

Whereas for compounds, PAB-108 and PAB-110, the dissociation constants were calculated by solving equations (4) and (5) for all the points below and above $\overline{n_{H}}=1$ respectively.

$$
\begin{gathered}
\log p K_{1}^{H}=p H+\log \left(\overline{n_{H}} /\left(\overline{n_{H}}-1\right)\right) \\
\log p K_{2}^{H}=p H+\log \left[\left(\overline{n_{H}}-1\right) /\left(2-\overline{n_{H}}\right)\right]
\end{gathered}
$$

For half-integral method, at $\overline{n_{H}}=0.5$ value and $\overline{n_{H}}=1.5, \mathrm{pK}_{1}^{\mathrm{H}}$ and $\mathrm{pK}_{2}^{\mathrm{H}}$ value were evaluated from

\begin{tabular}{|c|c|c|c|c|c|}
\hline \multirow[t]{2}{*}{ Comp. Code } & \multicolumn{2}{|c|}{$\mathrm{pK}_{1}^{\mathrm{H}}$} & & \multicolumn{2}{|c|}{$\mathrm{pK}_{1}^{\mathrm{H}} / \mathrm{pK}_{2}^{\mathrm{H}}$} \\
\hline & $\begin{array}{l}\text { Half-integral } \\
\text { method }\end{array}$ & $\begin{array}{c}\text { Average } \\
\text { method }\end{array}$ & & $\begin{array}{l}\text { Half-integral } \\
\text { method }\end{array}$ & $\begin{array}{l}\text { Average } \\
\text { method }\end{array}$ \\
\hline$P A B-101$ & 9.88 & 9.54 & $P A B-107$ & 10.90 & 10.90 \\
\hline$P A B-102$ & 12.78 & 12.33 & $P A B-108$ & $3.86^{*}$ & $3.88^{*}$ \\
\hline$P A B-103$ & 9.40 & 9.41 & & $10.2^{\$}$ & $9.73^{\$}$ \\
\hline$P A B-104$ & 9.50 & 9.50 & $P A B-109$ & 10.96 & 10.93 \\
\hline$P A B-105$ & 9.94 & 9.93 & $P A B-110$ & $4.00^{*}$ & $4.12^{*} 9.32^{\$}$ \\
\hline$P A B-106$ & 9.84 & 9.86 & & $9.8^{\$}$ & \\
\hline
\end{tabular}
the plot of $\overline{n_{H}}$ versus $p H$.

The evaluated dissociation constants by both average and half-integral methods are given in Table 2.

Table 2. The $\mathrm{pK}_{1}^{\mathrm{H}}$ and $\mathrm{pK}_{2}^{\mathrm{H}}$ values for compounds by different methods

*at $\overline{n_{H}}=1.5 ;$ Sat $\overline{n_{H}}=0.5$

Comparison of $\mathrm{pK}_{1}^{\mathrm{H}}$ and $\mathrm{pK}_{2}^{\mathrm{H}}$ values evaluated by two methods shows good agreement. Comparison of $p K_{1}^{H}$ values of studied compounds (except PAB-108 and PAB-110 which are having two replaceable hydrogens) shows that PAB-102 was most basic followed by PAB-109 while PAB-103 was most acidic.

All the compounds have the same central moiety but different side chains which affect the dissociation constant. PAB-102 contains cinnamaldehyde side chain which is found to increase the basic character of this compound whereas in PAB-109, due to furfuladehyde side chain, basic character is greater than other studied compounds but less than that of PAB-102. PAB-103 contains chloro group at meta position and its acidic character is higher. However, PAB-104 also contains chloro group but at para position and acidic character of this compound is slightly decreased. This suggests that the position of groups also affects the dissociation. In case of PAB-105, fluoro group is at para position which further decreases the acidic character in comparison to p-chloro group (as in PAB-104). Other compounds show intermediate acidic character. PAB-108 and PAB-110 contain hydroxyl groups at para positions and are having two replaceable hydrogens. There is very small difference in $\mathrm{p} K^{\mathrm{H}}$ values of these two compounds. However, considering the $\mathrm{pK}_{2}^{\mathrm{H}}$ values of these two (PAB-108 and PAB-110) compounds indicates the higher acidity of PAB-108 which may be due to the presence of methoxy group. Similar characteristic behavior was also reported earlier [24]. Alteration of hydroxy group with any other functional group 
changes the dissociation of compounds [25].

\section{Conclusion}

Out of ten studied compounds, two compounds; PAB-108 and PAB-110 have two displacable hydrogens and PAB-108 containing 3-methoxy and 4-hydroxy groups is more acidic than PAB-110 containing only 4-hydroxy group. Among remaining eight compounds, dissociation constant is maximum ( more acidic) for PAB-103 which is followed by PAB-104. PAB-102 exhibited minimum dissociation. The chloro group causes more dissociation as observed by PAB-103 and PAB-104 and its meta position increases dissociation slightly more than that in para position. The presence of cinnamaldehyde as in PAB-102 decreases dissociation. Thus, it can be concluded that type of substitution and their position in aromatic ring affects dissociation constant due to their inductive or mesomeric effects [26, 27].

\section{References}

1. R. Kumar, A. Mittal and U. Ramachandran, "Design and synthesis of 6-methyl-2-oxo-1,2,3,4-tetrahydropyrimidine-5- carboxylic acid derivatives as PPARY activators", Bioorg. Med. Chem. Lett., 2007, 17, 4613-4618.

2. R. L. Sawant and M. S. Bhatia, "Synthesis, screening and qsar studies of 3-formyl-2-oxo-1, 2, 3, 4-tetra hydropyrimidine analogues as antibacterial agents", Bull. Chem. Soc. Ethiopia, 2008, 22, 391-402.

3. M. K. Manal, M. R. Sameha, A. A. Mohamed, K. A. Eman and F. L. Phoebe, "Design, synthesis and cytotoxic activity of some novel compounds containing pyrazolo[3,4-d ]pyrimidine nucleus", Der Pharma Chem., 2013, 5, 109-124.

4. K. Weinhardt, M. B. Wallach and M. Marx, "Synthesis and antidepressant profiles of phenyl-substituted 2amino- and 2-[(alkoxycarbonyl)amino]-1,4,5,6-tetrahydro pyrimidines," J. Med. Chem., 1985, 28, 694-698.

5. A. Balkan, B. Tozkoparan, M. Ertan, Y. Sara and N. Ertekin, "New thiazolo [3, 2-a]pyrimidine derivatives, synthesis and calcium antagonistic activities," Boll. Chim. Farma., 1996, 135, 648-652.

6. K. Kaur and E. Knaus, "Synthesis of alkyl 6-methyl-4-(2-pyridyl)-1,2,3,4-tetrahydro-2H-pyrimidine-2-one-5carboxylates for evaluation as calcium channel antagonists," J. Heterocycl. Chem., 2007, 44, 745-747.

7. Y. Ji-Xin, C. Xiao-Qing, C. Di-Mei and H. Mao-Lin, "Synthesis, structure analysis, and antitumor activity of (R)-2,4-dioxo-5-fluoro-1(methoxycarbonyl) ethylamino carbonyl methyl] 1,2,3,4 tetrahydro pyrimidine," Chin. J. Chem., 2007, 25, 417-421.

8. V. Virsodia, R. R. S. Pissurlenkar, D. Manvar, C. Dholakia, P. Adlakha, A. Shah and E. V. Coutinho, "Synthesis, screening for antitubercular activity and 3D-QSAR studies of substituted $N$-phenyl-6-methyl-2-oxo-4phenyl-1,2,3,4-tetrahydro-pyrimidine-5-carboxamides" Eur. J. Med. Chem., 2008, 43, 2103-2115.

9. S. Mokale, S. Shinde, R. Elgire, J. Sangshetti and D. Shinde, "Synthesis and anti-inflammatory activity of some 3-(4,6-disubtituted 2 thioxo1,2,3,4 tetrahydro pyrimidin-5-yl)propanoic acid derivatives," Bioorg. Med. Chem. Lett., 2010, 20, 4424-4426.

10. R. Dhankar, A. M. Rahatgaonkar, R. Shukla, M. Chorghade and A. Tiwari, "Computer simulation of the in vitro and in vivo anti- inflammatory activities of dihydropyrimidines acid derivatives through the inhibition of cyclooxygenase-2", Med. Chem. Res., 2013, 22, 2493-2504.

11. K. Satyavathi, K. T. Naga Ravi, R. P. Bhoja and M. Sharmila, "Synthesis and screening of 3-formyl-2-thio1,2,3,4-tetrahydro pyrimidine analogues as antibacterial agents," Asian J. Chem., 2010, 22, 5182-5186.

12. S. N. Darandale, D. N. Pansare, N. A. Mulla and D. B. Shinde, "Green synthesis of tetrahydropyrimidine analogues and evaluation of their antimicrobial activity", Bioorg. Med. Chem. Lett., 2013, 23, 2632-2635.

13. N. Arora and S. Pandeya, "Synthesis and analgesic activity of novel pyrimidine derivatives," Int. J. Pharma. Sci. Rev. Res., 2011, 11, 48-52.

14. H. S. Basavaraja, P. Basavaraj, M. Vijaykumar, M. M. Hussain and B. N. Chidananda, "Synthesis and antimicrobial screening of some substituted INH- and THPHM-linked pyrimidines," Ind. J. Heterocycl. Chem., 2011, 20, 237-240.

15. M. Mansouri, A. Movahedian, M. Rostami and A. Fassihi, "Synthesis and antioxidant evaluation of 4-(furan-2yl)-6-methyl-2-thioxo-1, 2, 3, 4-tetrahydropyrimidine-5-carboxylate esters", Res. Pharma. Sci., 2012, 7, 257-264. 
16. M. Andrasi, P. Buglyo L. Zekany and A. Gaspar, "A comparative study of capillary zone electrophoresis and pH-potentiometry for determination of dissociation constants," J. Pharma. Biomed. Ana., 2007, 44, 1040-1047.

17. T. Fengxiang, G. Zhongli, L. Daqiang, C. Haoyu and Z. Suying, "Dissociation constant and solubility of (S)-2Hydroxy-4-phenylbutyric Acid," J. Chem. Eng. Data, 2013, 58, 1265-1270.

18. A. Ahmed, B. Ahmed and I. T. Ahmed, "Acid dissociation constants mercapto benzazoles in aqueous-organic solvent mixtures," J. Chem. Eng. Data, 1996, 41, 787-790.

19. J. A. Riddick, W. B. Bunger and T. Sakano, "Organic Solvents: Physical Properties and Methods of Purification", 4th edition, John Wiley \& Sons Inc., USA, 1986.

20. M. Calvin and K. W. Wilson, "Stability of Chelate Compounds". J. Am. Chem. Soc., 1947, 67, 2003-2007.

21. J. Bjerrum, "Metal amine formation in aqueous solution". P Hasse and Son, Coppehagen, 1941.

22. L. G. Van Uitert and G. G. Hass, "Studies on coordination compounds. I. A method for determining thermodynamic equilibrium constants in mixed so1vent," J. Am. Chem. Soc., 1953, 75, 451-454.

23. H. Irving and H. S. Rossoti, "The calculation of formation curves of metal complexes from pH titration curves in mixed solvents," J. Chem. Soc., 1954, 12, 2094-2010.

24. J. Ephraim, S. Alegret, A. Mathuthu, M. Bicking, R. Malcolm and J. Marinsky, "A unified physicochemical description of the protonation and metal ion complexation equilibria of natural organic acids (humic and fulvic acids). 2. Influence of polyelectrolyte properties and functional group heterogeneity on the protonation equilibria of fulvic acid," Environ. Sci. Tech., 1986, 20, 354-366.

25. A. Yoda,"Structure-Activity relationships of cardiotonic steroids for the inhibition of sodium- and potassiumdependent adenosine triphosphatase I. Dissociation rate constants of various enzyme-cardiac glycoside complexes formed in the presence of magnesium and phosphate," Mol. Pharmaco., 1973, 9, 51-60.

26. A. El-Sonbati, A. El-Bindary, A. Shoair and R. Younes, "Stereochemistry of new nitrogen containing heterocyclic aldehyde. vii. Potentiometric, conductometric and thermodynamic studies of novel quinolineazodyes and their metal complexes with some transition metals," Chem. Pharma. Bull., 2001, 49, 1308-1313.

27. Y. M. Issa, O. E. Sherif and S. M., Abbas, "Chelation behavior of Ce(III), Th(IV), and UO (VI) with 5,7dihydroxy-6-formyl-2-methylbenzopyran-4-one Schiff bases," Monat. Chem., 1998, 129, 985-998. 